\title{
Management of Percutaneous Endoscopic Gastrostomy Site Gastric Ulcer in a Patient with an Esophageal Stricture and Hiatus Hernia
}

\author{
Clement C H Wu ${ }^{1}$, James W $\mathrm{Li}^{1}$, Keng Sin $\mathrm{Ng}^{2}$ and Daphne S Ang ${ }^{1}$ \\ Departments of ${ }^{1}$ Gastroenterology and Hepatology, ${ }^{2}$ Diagnostic Radiology, Changi General Hospital, Singapore
}

Percutaneous endoscopic gastrostomy (PEG) is commonly performed for feeding difficulties, in patients suffering from complications of nasopharyngeal carcinoma (NPC) and its treatment, namely radiotherapy and surgery. This case report describes the challenges in hemostasis and subsequent re-establishment of enteral access for feeding, in an elderly patient with a history of NPC, treated surgically, followed by radiotherapy, who presented with massive hematemesis following reinsertion of her PEG shortly after an accidental dislodgement. Her previous nasopharyngectomy, wide field radiation therapy, and radical neck dissection precluded nasogastric tube feeding, and the presence of a large hiatus hernia made reinsertion of a new PEG technically challenging. This case highlights the methods used to overcome the above challenges. Clin Endosc 2018;51:99-102

Key Words: Gastrostomy; Intubation, gastrointestinal; Hernia, hiatal; Ulcer; Enteral nutrition

\section{INTRODUCTION}

Nasopharyngeal carcinoma (NPC) is common in East Asia, ${ }^{1}$ with radiotherapy and surgery being the mainstays of treatment. ${ }^{2,3}$ These are associated with significant morbidity, ${ }^{4}$ including dysphagia and anatomical distortion, which can lead to feeding difficulties. Percutaneous endoscopic gastrostomy (PEG) feeding is commonly used for enteral nutrition, in patients with dysphagia from head and neck malignancies such as NPC., ${ }^{5,6}$ Ulcer formation might occur after PEG insertion $^{7,8}$ which might be associated with gastrointestinal bleeding or perforation. ${ }^{7-9}$

Most PEG-related ulcers occur at the base of intragastric bumper of the tube, due to pressure necrosis. ${ }^{6,8,10-12}$ This usually develops subacutely, occurring in an average of 1-18 months after PEG insertion. ${ }^{13}$ Gastric ulcers can also occur

Received: March 4, 2017 Revised: April 4, 2017

Accepted: April 26, 2017

Correspondence: James W Li

Department of Gastroenterology and Hepatology, Changi General Hospital, 2 Simei Street 3, 529889, Singapore

Tel: +65-6788-8833, Fax: +65-6788-0933, E-mail: james.li@mohh.com.sg

(cc) This is an Open Access article distributed under the terms of the Creative Commons Attribution Non-Commercial License (http://creativecommons.org/ licenses/by-nc/3.0) which permits unrestricted non-commercial use, distribution, and reproduction in any medium, provided the original work is properly cited. opposite to the PEG insertion site.,8

\section{CASE REPORT}

We describe the case of a 72-year-old female with a large hiatus hernia, who presented to our center with massive hematemesis after reinsertion of a dislodged PEG tube. She had a history of NPC with multiple recurrences, and underwent wide field radiation therapy, left radical neck dissection and a left nasopharyngectomy. The patient was on long term feeding via a 20 -French PEG tube. She had earlier visited another center with a dislodged PEG, which had last been changed 6 months before the dislodgement. A new PEG was reinserted at the Accident and Emergency Department and the patient was discharged on the same day.

However, she presented 2 days later at our center, with large volume hematemesis accompanied by blood clots. The patient was intubated for airway protection as she had a low Glasgow Coma Scale score at the time of presentation. Additionally, there was significant blood loss requiring transfusion. Both, a therapeutic gastroscope and a standard gastroscope could not cross the tight upper esophageal sphincter (UES) due to scarring from previous radiotherapy. An Olympus 


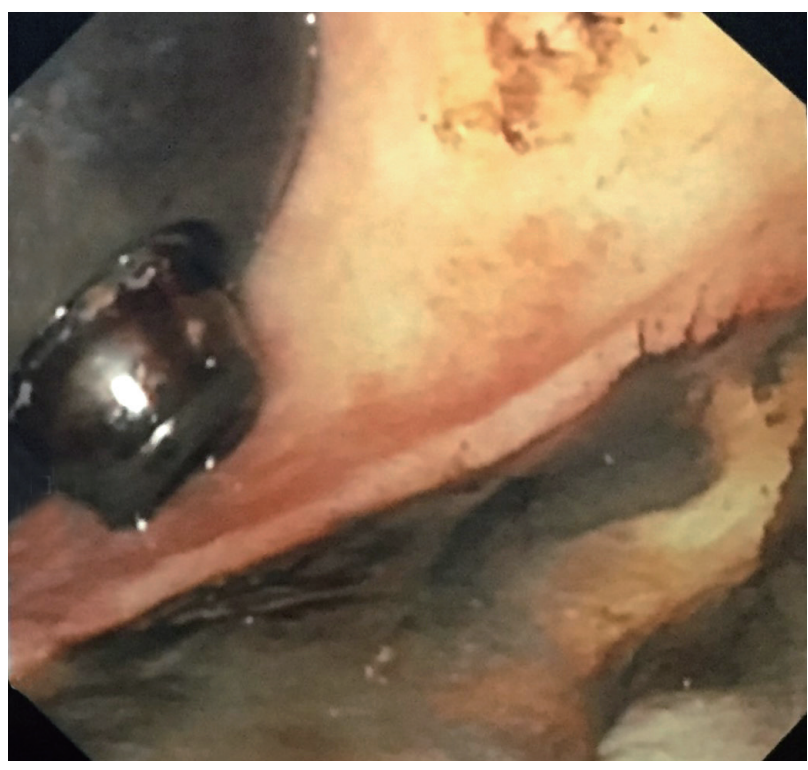

Fig. 1. Gastroscopic view showing Forrest llb ulcer located at the antrum opposite the tip of the percutaneous endoscopic gastrostomy tube.

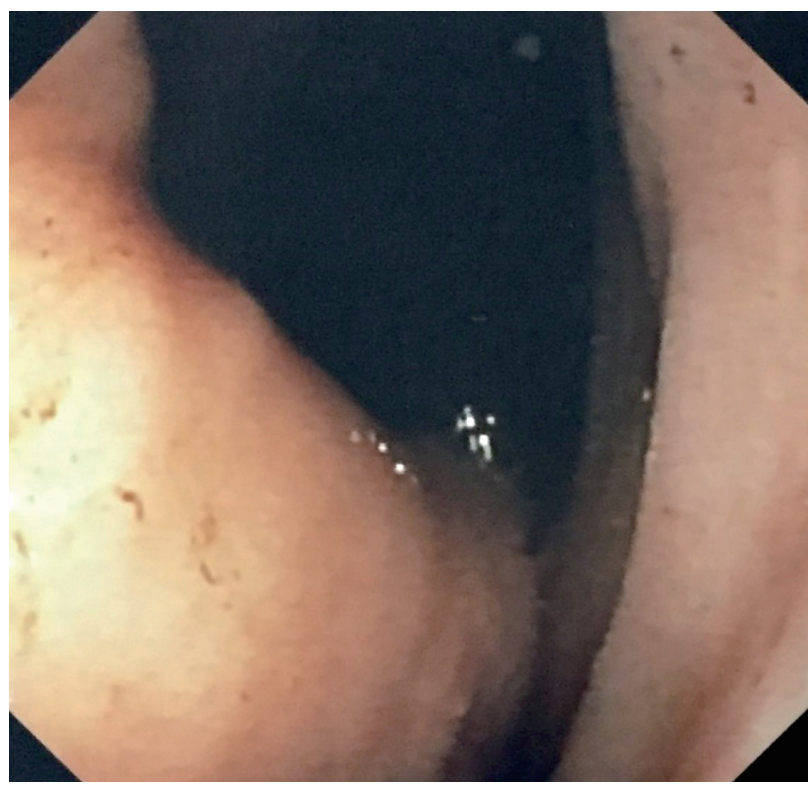

Fig. 2. Retroflex view on gastroscopy demonstrating tight gastric space secondary to hiatus hernia, with most of the corpus located above the diaphragm.

GIF-XP190N pediatric gastroscope (Olympus Corporation, Tokyo, Japan) was eventually used to traverse the stricture.

During endoscopy, the tip of the PEG tube was seen abutting the opposite antral wall just adjacent to the pylorus, resulting in a large Forrest IIb ulcer (Fig. 1). A large hiatus hernia $(20.2 \times 11.9 \mathrm{~cm})$ was also noted (Fig. 2). Therapeutic options were limited, given the smaller working channel of the pediatric gastroscope. Removal of the adherent blood clot and hemoclip application could not be performed. A total of $7 \mathrm{~mL}$ of adrenaline was injected into the ulcer edges to

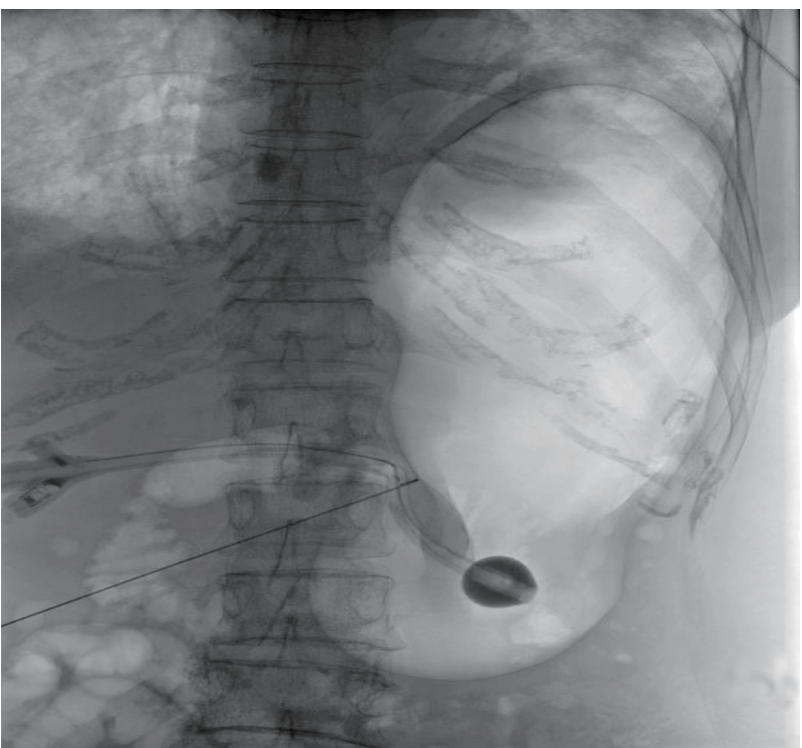

Fig. 3. Stomach insufflation via existing the percutaneous endoscopic gastrostomy tube prior to tube change. The gastric fundus and most of the corpus is located within the intrathoracic space.

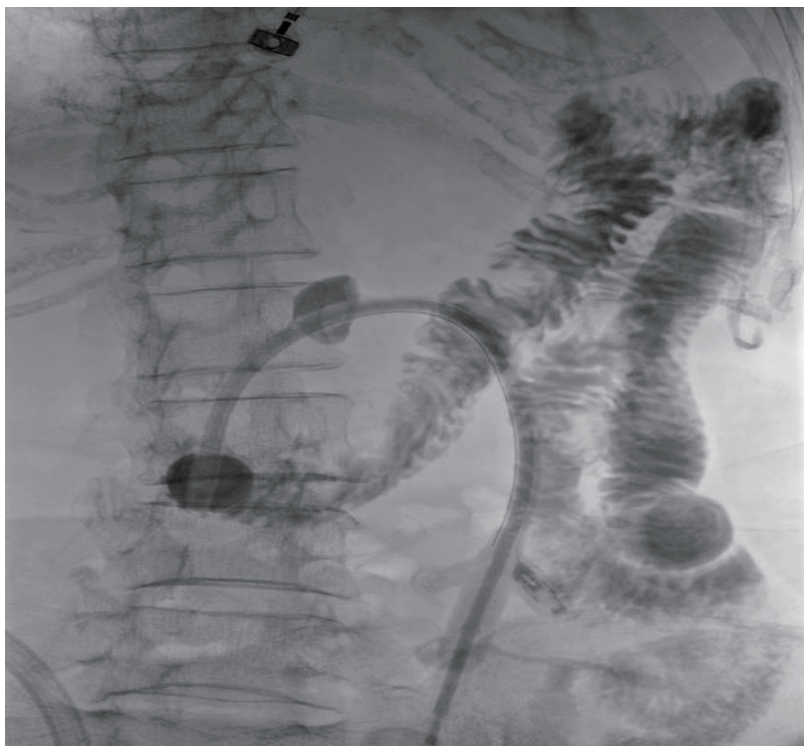

Fig. 4. An interim 20-Fr low profile percutaneous endoscopic gastrostomy tube was inserted via the existing tract, with the tip placed in the duodenum.

secure hemostasis.

During fluoroscopy, most of the gastric corpus and fundus was found to be in the thorax (Fig. 3). As a result, there was very limited space in the gastric antrum and pylorus for a new PEG tube insertion. The existing PEG tube was removed to allow healing of the ulcer, and a 16-French Foley's catheter was inserted by an interventional radiologist under fluoroscopic guidance, to maintain patency of the PEG tract. Subsequently, an interim 20-French low profile PEG tube (Kimberly Clark Corporation, Irving, TX, USA) was insert- 


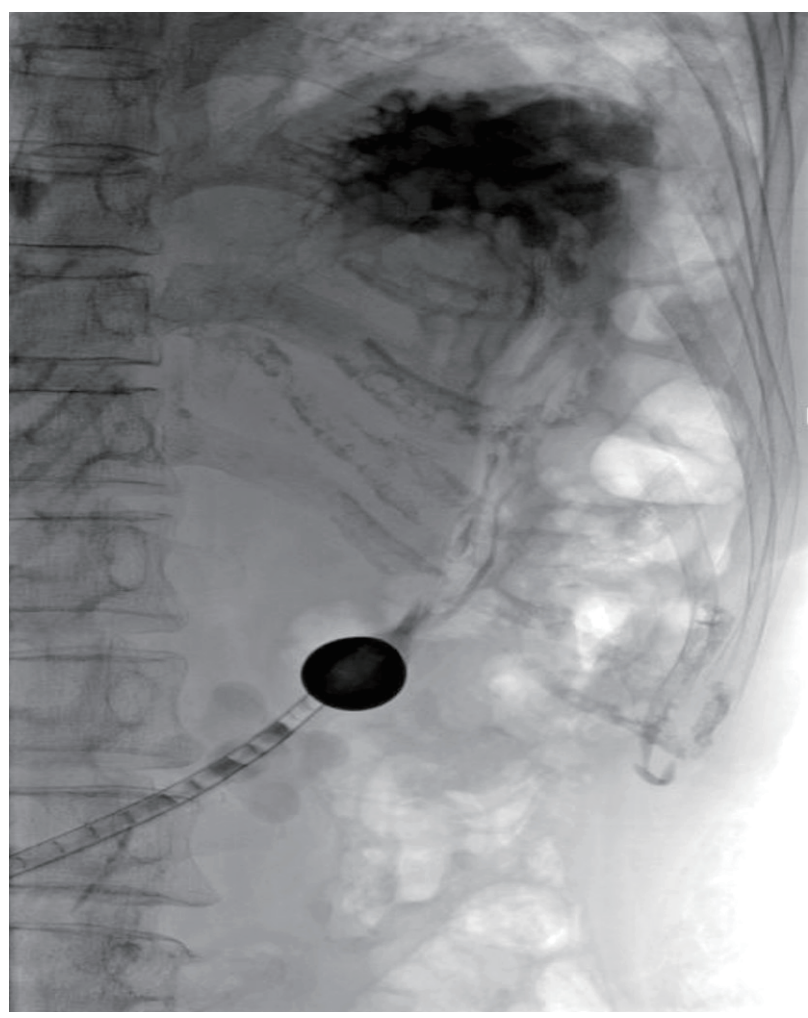

Fig. 5. A new 20-Fr percutaneous endoscopic gastrostomy tube was inserted at a different anatomical site under fluoroscopic guidance.

ed through the existing tract, 5 days later. The tip of the new PEG tube was placed in the duodenum, to aid healing of the gastric ulcer and enteral feeding recommenced (Fig. 4). In addition, the patient was administered Esomeprazole $40 \mathrm{mg}$ twice daily, through the feeding tube, to promote ulcer healing. Three weeks later, reinsertion of a new 20-French PEG tube at a different location in the pyloric antrum, was successfully performed (Fig. 5). The procedure was performed by insufflating air through the existing PEG, which allowed adequate visualization of the intra-abdominal gastric lumen despite the large hiatus hernia. The new PEG was inserted via a separate tract, under radiological guidance, despite the narrow working space available due to the hiatus hernia. The patient tolerated the procedures without any further complications, and was discharged shortly thereafter.

\section{DISCUSSION}

A hiatus hernia involves protrusion of the upper part of stomach into the thoracic cavity due to a diaphragmatic defect, thus reducing the intra-abdominal component of the stomach. ${ }^{14}$ Ideally, a PEG should be inserted into the gastric body. However, our patient had a large hiatus hernia, leading to a very small window available for creating a PEG tube insertion site. The original PEG insertion site was in the gastric antrum and this was likely to be due to anatomical limitations posed by the hiatus hernia. Over time, the close proximity of the PEG tip to the opposite antral wall next to the pylorus predisposed it to erosions, resulting in an iatrogenic ulcer. Following hemostasis, reinsertion of a new PEG tube was delayed to allow time for ulcer healing.

There were limited interim feeding options for our patient. Nasogastric tube insertion was impossible due to anatomical distortion of the nasopharynx from the patient's previous nasopharyngectomy. Orogastric tube insertion was also challenging, given the presence of a tight UES stricture, and although technically feasible, would have severely compromised the patient's quality of life. Re-establishment of enteral feeding via a new PEG, was the most viable option for longterm feeding in this patient, who was otherwise well, before the present complication.

Re-establishing enteral feeding through a percutaneous endoscopic transgastric jejunostomy tube via the existing site, could have avoided the risks and challenges of re-inserting the PEG at a different anatomical location. However, enteral feeding through a jejunostomy requires the use of a pump and continuous feeding initially, and the patient might not tolerate eventual escalation to bolus feeding. This could have an adverse effect on the patient's quality of life. Even if the patient is able to tolerate progression to bolus feeding, the boluses have to be given at lower volumes and higher frequencies than via the gastric route. A continuous feeding pump may also be required to deliver the remaining calories at night when the patient is sleeping, resulting in increased healthcare costs to the patient, and increased caregiver burden.

Re-insertion of another PEG tube through the same tract carried the risk of recurrent ulcer bleeding and perforation due to the tight anatomical space in the antrum as described above. Moreover, the partially healed antral ulcer presented an existing mucosal defect directly opposite the tip of the PEG if the same tract were used. Although previous studies have described the use of a low profile PEG (a PEG tube with minimal protrusion of the feeding tube beyond the intra-gastric balloon, to minimize its intra-gastric profile) or reinsertion at an alternative site, ${ }^{7,13,15}$ they were not performed in patients with a large hiatus hernia due to a limited working space as in this case.

Insertion of a new PEG at a different anatomical site during index endoscopy for hemostasis was not possible, as the patient was not stable. By inserting a temporary Foley's catheter under fluoroscopic guidance and directing the tip into the duodenum, we were able to continuously feed our patient and relieve the erosive pressure effect on the ulcer. This al- 
lowed time for the ulcer to heal and the patient's condition to stabilize, before a new PEG insertion was planned. We used an adequately large Foley's catheter in order to prevent leakage of gastric juices around the gastrostomy site. Although maintaining the Foley's catheter in situ while awaiting definitive PEG resiting would have been a safe and effective option to maintain enteral feeding, our patient was severely deconditioned during her hospital stay, and an interim low profile PEG made nursing care easier and allowed our patient to participate in physiotherapy and rehabilitative exercises. The definitive PEG resiting procedure was anticipated to be complex given her known anatomical distortions, and having reliable enteral access enabled her to receive adequate nutrition and permitted active participation in physiotherapy. This was crucial in expediting the optimization of her nutritional and functional status.

When a new PEG was inserted through the existing tract in the interim, the tip was placed in the duodenum and confirmed radiologically. This prevented further erosion of the existing antral ulcer until insertion of a PEG via a new tract could be performed. Considering the multiple challenges for PEG insertion via a new tract in this patient, a fluoroscopically guided approach was deemed most appropriate. The low profile PEG was used to insufflate air into the stomach. This expanded the working space to allow better visualization of the intra-abdominal gastric lumen during insertion of the new PEG under radiological guidance, thereby overcoming the technical difficulties posed by the large hiatus hernia and the presence of a large antral ulcer.

Our case highlights the challenges faced during PEG insertion in patients with a large hiatus hernia. The distorted anatomy increases the risk of tube malposition and iatrogenic ulceration. Fluoroscopic guided insertion in the setting of a distorted gastric anatomy might be a safer option, including the use of a low profile PEG tube to reduce the risk of mechanical injury. In the event of severe bleeding from a gastric ulcer secondary to a poorly positioned PEG, a temporary large bore Foley's catheter inserted through the same gastrostomy tract will allow feeding to continue and the ulcer to heal.
Conflicts of Interest

The authors have no financial conflicts of interest.

\section{REFERENCES}

1. Wei WI, Sham JS. Nasopharyngeal carcinoma. Lancet 2005;365:20412054.

2. Lee AW, Sze WM, Au JS, et al. Treatment results for nasopharyngeal carcinoma in the modern era: the Hong Kong experience. Int J Radiat Oncol Biol Phys 2005;61:1107-1116.

3. Tham IW, Hee SW, Yeo RM, et al. Treatment of nasopharyngeal carcinoma using intensity-modulated radiotherapy-the national cancer centre Singapore experience. Int J Radiat Oncol Biol Phys 2009;75:14811486.

4. Yen TT, Lin CH, Jiang RS, Shih YT, Yen HR, Liang KL. Incidence of late-onset pneumonia in patients after treatment with radiotherapy for nasopharyngeal carcinoma: a nationwide population-based study. Head Neck 2015;37:1756-1761.

5. Luman W, Kwek KR, Loi KL, Chiam MA, Cheung WK, Ng HS. Percutaneous endoscopic gastrostomy--indications and outcome of our experience at the Singapore general hospital. Singapore Med J 2001;42:460465.

6. Hede GW, Faxén-Irving G, Olin AÖ, Ebbeskog B, Crisby M. Nutritional assessment and post-procedural complications in older stroke patients after insertion of percutaneous endoscopic gastrostomy - a retrospective study. Food Nutr Res 2016;60:30456.

7. Delatore J, Boylan JJ. Bleeding gastric ulcer: a complication from gastrostomy tube replacement. Gastrointest Endosc 2000;51(4 Pt 1):482-484.

8. Kanie J, Akatsu H, Suzuki Y, Shimokata H, Iguchi A. Mechanism of the development of gastric ulcer after percutaneous endoscopic gastrostomy. Endoscopy 2002;34:480-482.

9. Chong C, Derigo L, Brown D. Massive gastric bleeding: a rarely seen subacute complication of percutaneous endoscopic gastrostomy. Intern Med J 2007;37:787-788.

10. Wiener GJ. Complication caused by the tip of gastrostomy tubes and foley catheters. Am J Gastroenterol 1999;94:3656-3657.

11. Nishiguchi Y, Fuyuhiro Y, Lee JT, et al. Percutaneous endoscopic gastrostomy, duodenostomy and jejunostomy. Diagn Ther Endosc 1994;1:37-43.

12. Spiegelman G, Goldberg RI. Gastric ulceration following PEG replacement. Gastrointest Endosc 1992;38:397-398.

13. Mahadeva S, Chua CJ, Malik A, Chin KT, Jeyasingam R. Jatrogenic gastric ulceration in patients with percutaneous endoscopic gastrostomy feeding. Intern Med J 2007;37:732-733.

14. Xenos ES. Percutaneous endoscopic gastrostomy in a patient with a large hiatal hernia using laparoscopy. JSLS 2000;4:231-233.

15. Ulla JL, Alvarez V, Carpio D, Ledo L, Vázquez-Sanluis J, Vázquez-Astray E. Upper gastrointestinal bleeding in a patient with a balloon bumper PEG feeding tube. Surg Laparosc Endosc Percutan Tech 2005;15:94. 